\title{
Effect of Composite Spinneret Systems on the Formation of Helical Nanofibers via Electrospinning
}

\author{
Wenhua Ding ${ }^{1, a}$, Huihui $\mathrm{Wu}^{1, \mathrm{~b}}$, and Yongchun Zeng ${ }^{1, \mathrm{c}}$ \\ ${ }^{1}$ College of textiles, Donghua University, Shanghai, 201620, China \\ ${ }^{2}$ College of textiles, Donghua University, Shanghai, 201620, China \\ ${ }^{3}$ College of textiles, Donghua University, Shanghai, 201620, China \\ a18703603920@163.com, b1129019@mail.dhu.edu.cn, cyongchun@dhu.edu.cn
}

Key words: Composite spinneret. Electrospinning. Helical nanofibers

Abstract: In this work, we report an approach to prepare helical nanofibers via co-electrospinning. The results of the experiment demonstrate that Composite spinneret is necessary for the formation of helical fibers. The off-centered system could produce more uniform helical structures. In order to explore the effect of contact time of the core-shell solutions to form helical nanofibers, three kinds of spinnerets with different structures are compared. The contact time influence the degree of compatibility of the components. Suitable compatibility is conducive to the formation of helical nanofibers.

\section{Introduction}

Nanofibers with different structures can be obtained by electrospinning[1]. Compared with the conventional electrospinning, composite spinneret is a sample approach for prepared composite nanofibers with two polymers. Composite spinneret includes coaxial, off-centered and sidy-byside[2]. Nanofibers with helical structure not only to maintain the high specific surface area and high porosity, but also added some excellent properties: helical nanofibers have greater elongation, three-dimensional helical structure provides more cavity, it has a higher porosity. These unique properties suggests a extensive applications such as opt-electronics, filters or textiles etc. Two polymers with different properties can be prepared helical nanofibers such as different electrical conductivity of the polymers, different shrinkage of the polymers. Chen et al found an effective method for the preparation of helical nanofibers via composite spinneret. They combined a thermoplastic elastomer polyurethane (TPU) component and a rigid component[3]. Lin et al combined thermoplastic polymer and elastomeric polymer produce helical nanofibers by a side-byside spinneret[4]. In this work, we report an method to obtain helical nanofibers via two polymers with different shrinkage. Experimental work is made to explore the spinneret structure on the formation of helical nanofibers by composite spinneret.

\section{Experimental}

Material Preparation.Poly(m-phenylene isophthalamide) (Nomex) were purchased from xiangrun Trading Co., Ltd., China. TPU was from Byer Materials Science, Germany. N,N,dimethylacetamide(DMAc), N,N,-dimethylformamide(DMF), tetrahydrofuran(THF), anhydrous lithium chloride( $\mathrm{LiCl})$ were purchased from Shanghai Lingfeng Chemical Reagents Co., Ltd., China. All materials were used without further purification.

Nomex solutions with the concentrations $12 \mathrm{wt} \%$ were prepared by dissolving the chopped Nomex in DMAc with $1.8 \mathrm{wt} \% \mathrm{LiCl}$. The solutions were stirred for $24 \mathrm{~h}$ at $100^{\circ} \mathrm{C}$. TPU solutions were prepared by dissolving TPU pellets in mixture solvents of $\mathrm{V}_{\mathrm{DMF}} / \mathrm{V}_{\mathrm{THF}}=3: 1$, stirred $5 \mathrm{~h}$ at ambient temperature.

Experimental Setup.The schematic of the co-electrospinning system is shown in Figure 1(a). The solutions of two solutions were separately fed into the spinneret via corresponding syringes and pumps. A high-voltage supply was applied to the spinneret and the collector. The structure of the spinnerets are shown in Figure 1(b). 


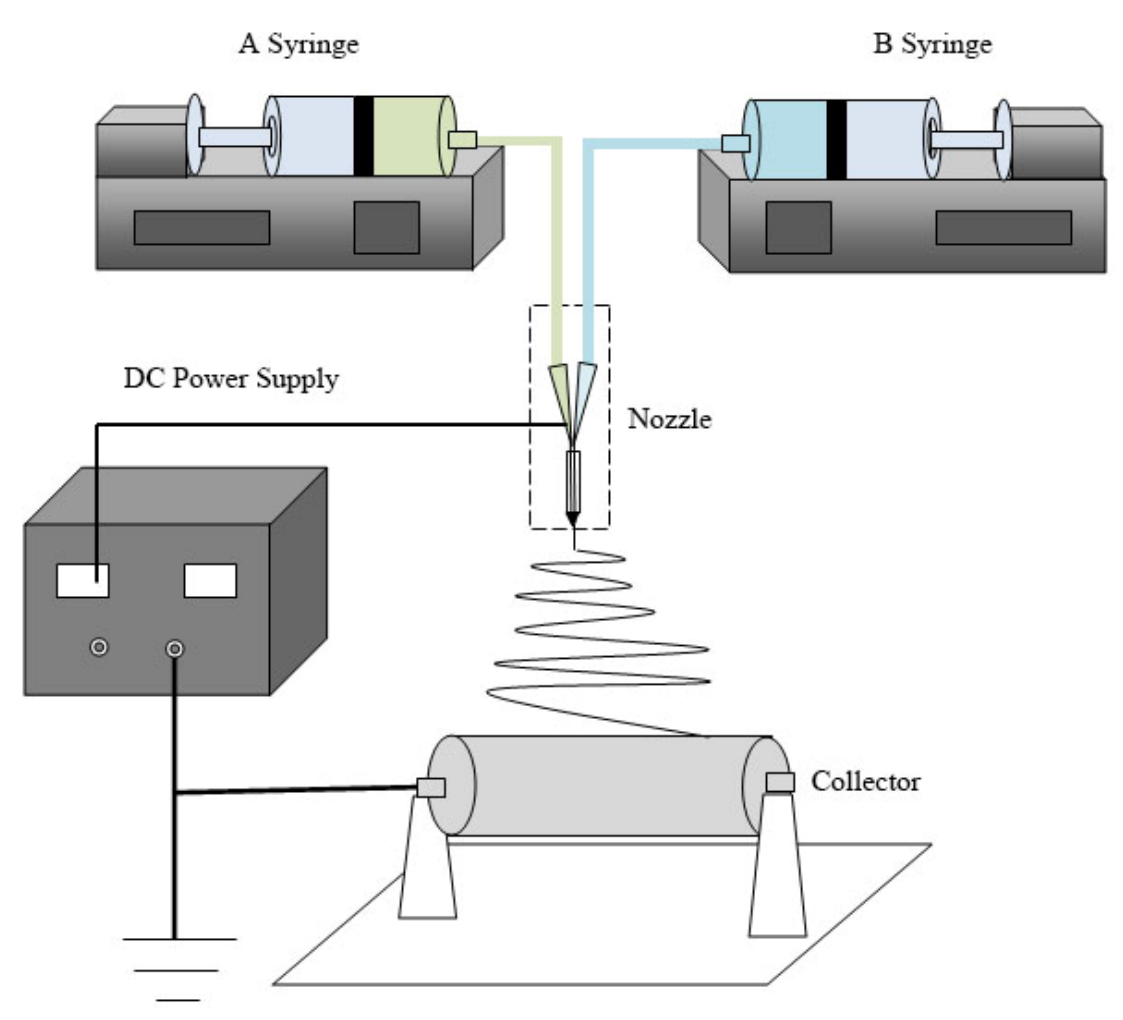

(a)
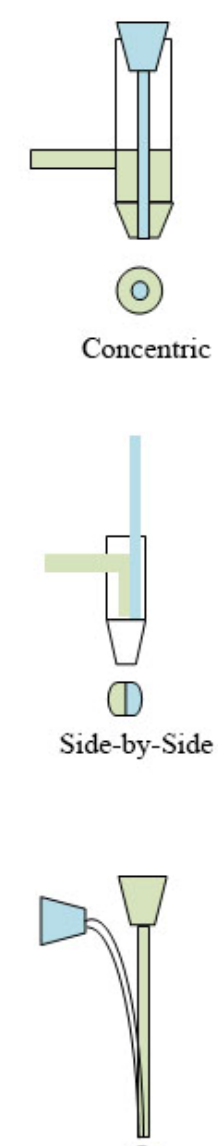

(2)

Off-centered

(b)

Figure.1 Schematic of co-electrospinning system: (a) experimental setup (b) structure of spinnerets. Characterization.The morphology of the resultant fibers were observed under Scanning Electron Microscope(SEM) (TM3000) after gold coating. The average fiber diameter and helical coil diameter were calculated from SEM images using Image J software from a collection of 50 fibers.

\section{Results and discussion}

The fibers were generated by electrospinning $12 \mathrm{wt} \%$ Nomex in DMAc with $1.8 \mathrm{wt} \% \mathrm{LiCl}$ and 18 wt $\%$ TPU in $\mathrm{V}_{\mathrm{DMF}} / \mathrm{V}_{\mathrm{THF}}=3: 1$. The resultant fibers of Nomex/TPU prepared by coaxial, side-by-side and off-centered are shown in Figure 2. Also shown is the result of single needle system, where the two solutions were mixed into the single needle. The helical fibers can be observed from the three kinds of composite spinneret systems. On the contrary, the single needle system gave only straight fibers. Therefore, the composite spinneret system is necessary for producing helical fibers. It is obvious that helical fibers can be fabricated effectively by off-centered system. The coaxial spinneret produces a few helical fibers. The average fiber diameters prepared by coaxial spinneret, side-by-side and off-centered were $331 \mathrm{~nm}, 345 \mathrm{~nm}$ and $266 \mathrm{~nm}$, respectively. The helical coil diameters were $920 \mathrm{~nm}, 802 \mathrm{~nm}$ and $896 \mathrm{~nm}$ accordingly. 

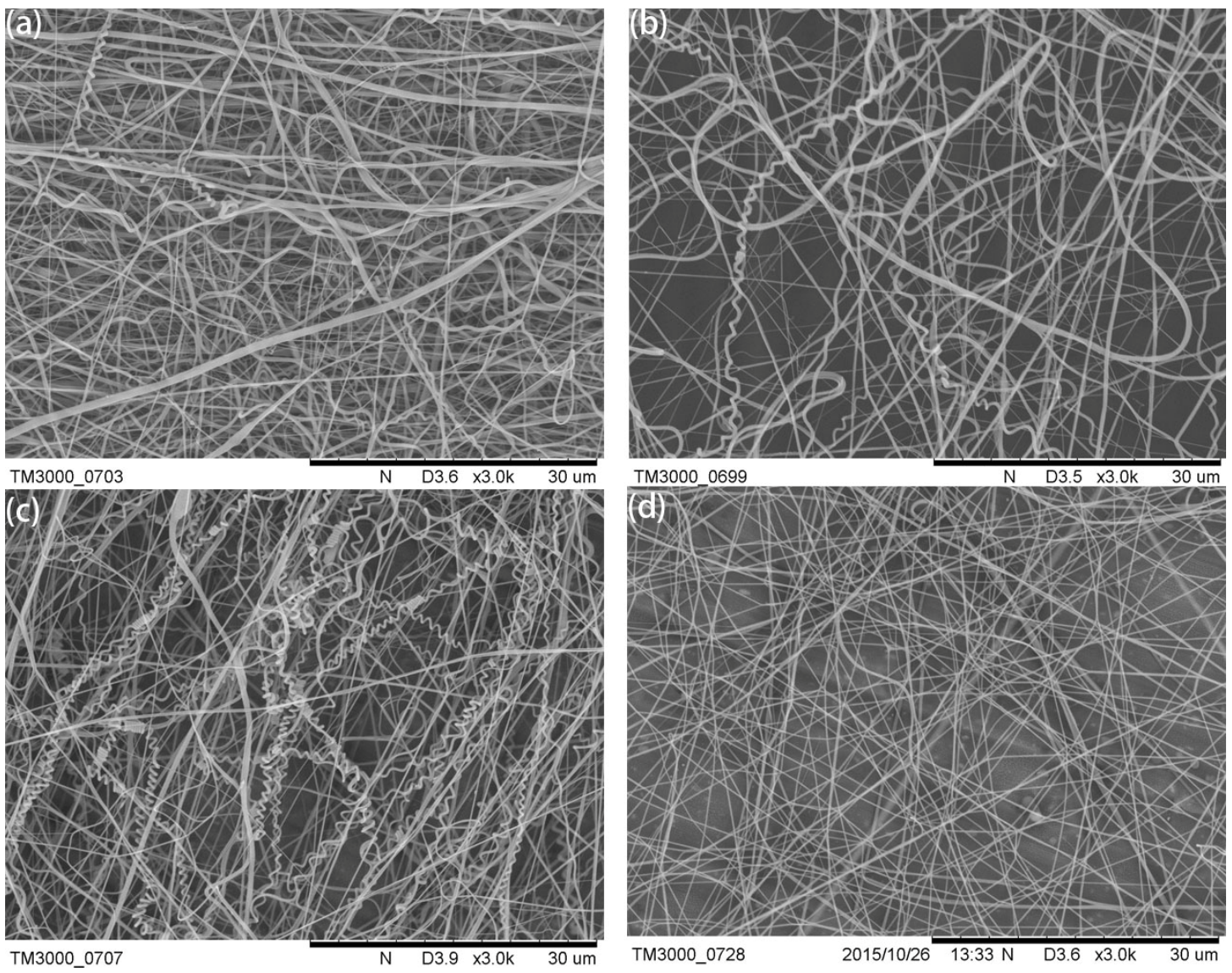

Fig.2 SEM images prepared by different spinneret: (a) coaxial (b) off-centered (c) side-by-side (d) single needle.

It is observed that when the two solutions were mixed uniformly, helical fibers are difficult to be fabricated and only a few spirals were prepared by the side-by-side system. We think the contact time of two polymer solutions have a greater influence on the formation of helical nanofibers. To further explore effect of the contact time of the two solutions on the helical fiber formation, the offcentered spinneret is improved. As shown in Figure 3(a), the spinneret with a retractive core-needle was made by retracting the core needle of the off-centered spinneret. Accordingly, the spinneret with an extended core-needle was shown in Figure3(c). The core needle is not blunt in these spinnerets. Two steel capillary were used to deliver the polymer solutions. The fibers were generated by electrospinning $12 \mathrm{wt} \%$ Nomex in DMAc with $1.8 \mathrm{wt} \% \mathrm{LiCl}$ and $18 \mathrm{wt} \% \mathrm{TPU}$ in $V_{\mathrm{DMF}} / \mathrm{V}_{\mathrm{THF}}=3: 1$. Figure 3 illustrates droplet contact diagrams and corresponding SEM images. 
(a)

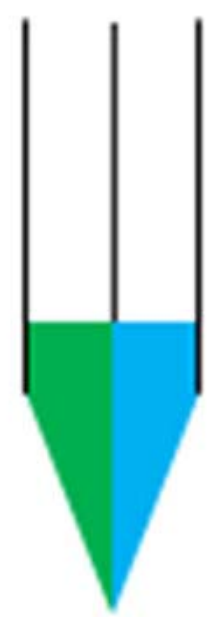

(b)

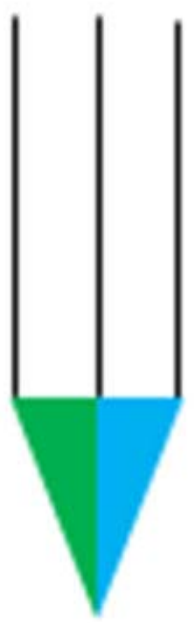

(c)

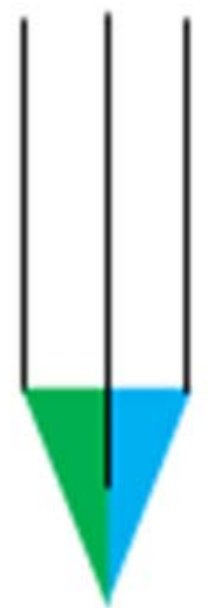

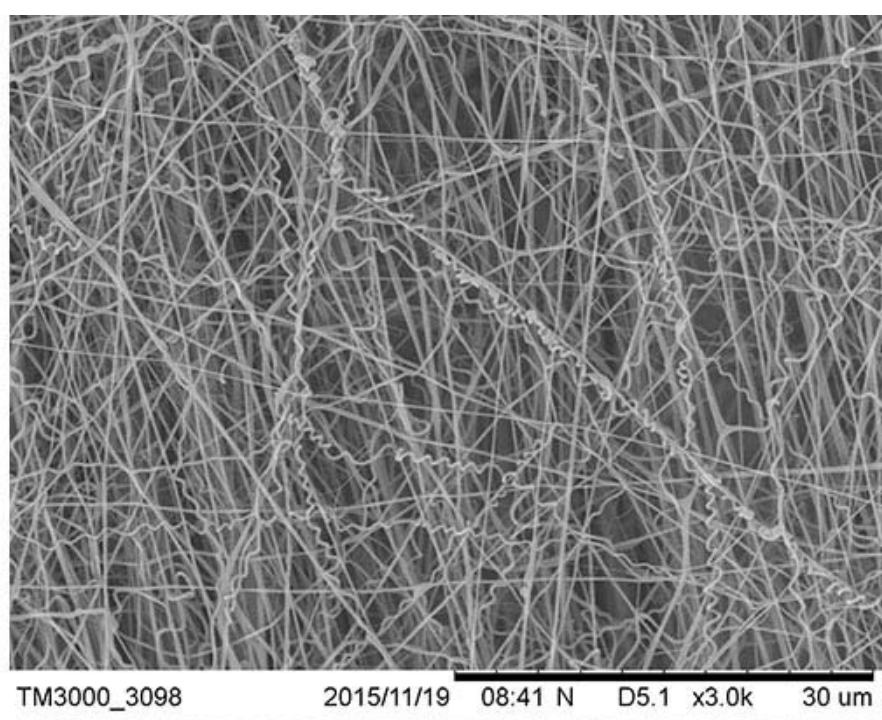
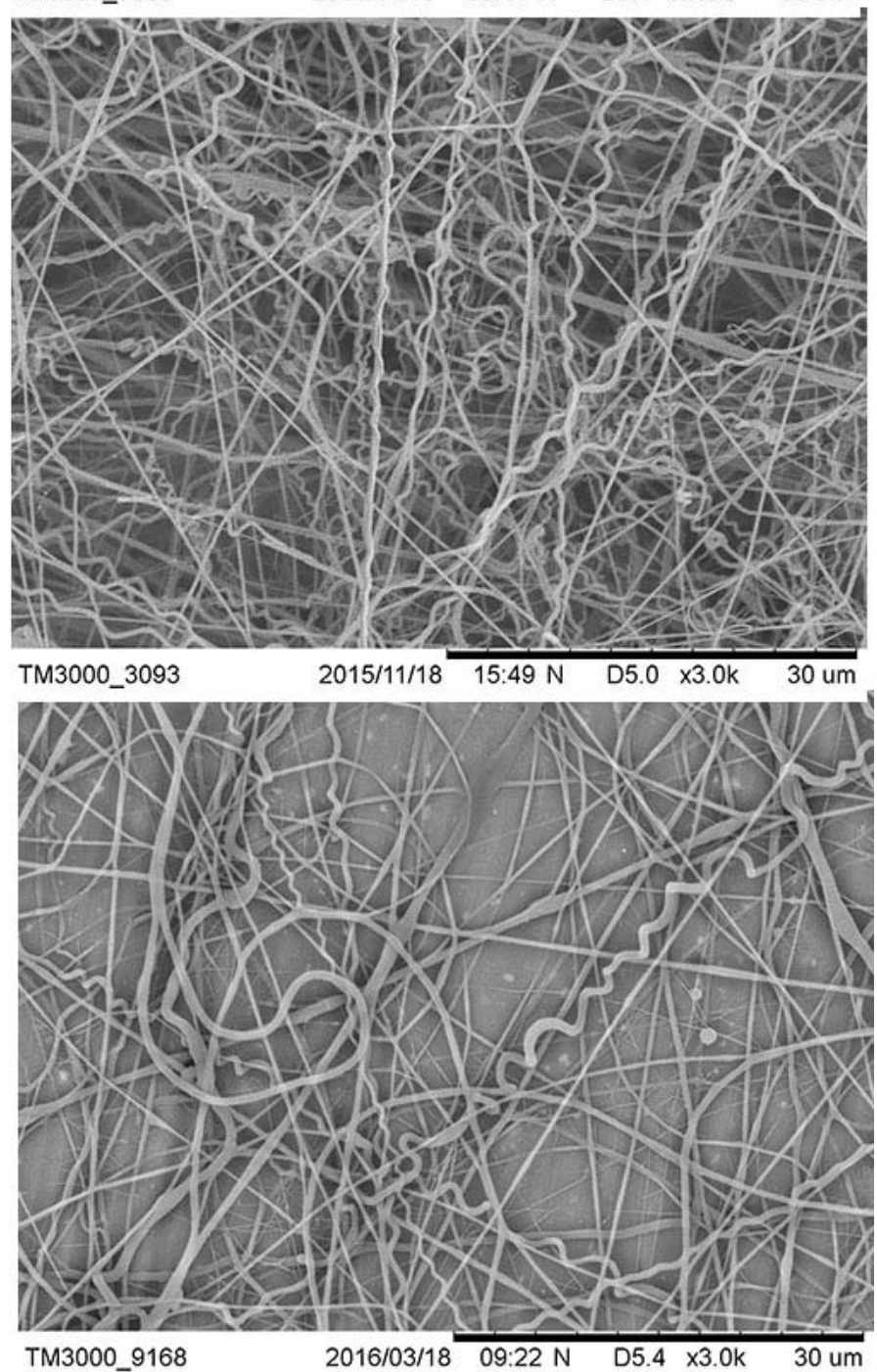

Figure.3 Droplet contact diagrams and corresponding SEM images: (a) a spinneret spinneret with a retractive core-needle (b) normal off-centered spinneret (c) a spinneret with an extended coreneedle.

Although the helical structures can be observed from three kinds of spinnerets, the efficiency of the helical nanofiber formation was greatly different. We ascribe the different efficiency to the different contact time of the two polymer droplets. Figure 3 show the SEM image of the helical nanofibers obtained by the three spinnerets. It can be found that some heilical nanofibers were prepared (Figure 3a). A lot of fibers with helical can be obtained by the normal off-centered 
spinneret (Figure 3b), while only a few helical fibers can be prepared by the spinneret with an extended core-needle (Figure 3c). Two polymer solutions have different contact time with different spinnerets in co-electrospinning. When the spinneret with a retractive core-needle is used during the co-electrospinning, the solutions of the two polymer solutions contact each other closely. The interaction between the two components is small in the electric field. It is not conducive to obtain the helical structure. As to the normal off-centered spinneret, the two polymer solutions contact at the outlet of the spinneret. The two solutions cannot contact effectively, leading to a larger interaction between the two components, which favors helical nanofibers. Two polymer solutions contact at the end of the Taylor cone in Figure 3(c), result in a short contact time of the two solutions. When they are drawn in the electric field, only a few solution achieve miscible. The contact time influence the degree of compatibility of the components. Suitable compatibility is conducive to the formation of helical nanofibers.

\section{Conclusions}

Three composite spinneret electrospinning systems, the coaxial, the side-by-side and the offcentered systems, are studied and compared with the single-needle system. The results of the experiments demonstrate that co-spinneret is necessary for the formation of helical fibers. The offcentered system seems to produce more uniform helical structures. In order to explore the effect of contact time of the two solutions on the helical fiber formation, three kinds of spinnerets are compared. The contact time influence the degree of compatibility of the components. Suitable compatibility is conducive to the formation of helical nanofibers.

\section{Acknowledgment}

This work was financially supported by the National Natural Science Foundation of China (11272088), the Fundamental Research Funds for the Central Universities.

\section{References}

[1] N. Fedorova, B. Pourdeyhimi: Journal of Applied Polymer Science, 2007, 104: 3434-3442.

[2] S. Chen, H. Hou, P. Hu, J.H. Wendorff, A. Greiner, S. Agarwal: Macromolecular Materials And Engineering, 2009, 294, 781-786.

[3] S. Chen, H. Hou, P. Hu, J.H. Wendorff, A. Greiner, S. Agarwal: Macromolecular Materials and Engineering, 2009, 294, 265-271.

[4] T. Lin, H. Wang, X. Wang: Advance Materials, 2005, 17: 2699-2703. 